\title{
The impact of lesion location and lesion size on post-stroke infection frequency
}

\author{
Jens Minnerup, $\mathrm{MD}^{1 *}$; Heike Wersching, MD ${ }^{1 *}$; Benjamin Brokinkel, \\ Rainer Dziewas, MD ${ }^{1}$; Peter Ulrich Heuschmann, MD²; \\ Darius Günther Nabavi, MD³; Erich Bernd Ringelstein, MD'; \\ Wolf-Rüdiger Schäbitz, MD ${ }^{4}$ Martin Andreas Ritter, MD ${ }^{1}$
}

1 Department of Neurology, University of Münster, Albert-Schweitzer-Straße 33, 48149 Münster, Germany.

2 Center for Stroke Research Berlin, Charité-Universitätsmedizin, Charitéplatz 1, 10117 Berlin, Germany.

3 Department of Neurology, Vivantes Klinikum Neukölln, Rudower Straße 48, 12351 Berlin, Germany.

4 Department of Neurology, University of Münster and Evangelisches Krankenhaus Bielefeld, Burgsteig 13, 33617 Bielefeld, Germany.

* These authors contributed equally to this work

Address correspondence:

Jens Minnerup (Universitätsklinikum Münster, Klinik und Poliklinik für Neurologie, AlbertSchweitzer-Straße 33, 48149 Münster, Germany, Phone: 49-251-83-48196, FAX.: 49-25183-48181, E-mail: minnerup@uni-muenster.de)

Keywords: Stroke, infection, stroke induced immunosuppression, pneumonia

Word count: 2501 


\begin{abstract}
Objectives: Infections in stroke patients are common and significantly affect outcome. Various predictors of post-stroke infections were determined, such as degree of neurological impairment and implementation of therapeutic interventions. We investigated whether stroke location and stroke size are independent risk factors for post-stroke infections.

Methods: Five hundred ninety-one patients with acute stroke who were treated on our stroke unit were included in a prospective observational study. Pre-defined endpoints were pneumonia, urinay tract infection (UTI) and other infections. The odds ratio (OR) of infections was calculated for various stroke locations, stroke lateralization and three categories of stroke size. Logistic regression models were used to adjust for factors significantly associated with post-stroke infections in single factor analysis.

Results: In single factor analysis the left anterior cerebral artery territory was associated with pneumonia. After adjustment for relevant covariates this association was no longer statistically significant. Stroke lateralization showed no association with infection frequency. The largest stroke size was positively associated with pneumonia (OR $3.5, P<0.001)$. The smallest lesion size was significantly less associated with the occurrence of UTI (OR 0.4 , $\mathrm{P}<0.01)$
\end{abstract}

Conclusion: In our study lesion size is an independent risk factor for the development of post-stroke infection. Particular brain regions associated with infections could not be determined. 
Mortality and neurological outcome of stroke patients are crucially influenced by the occurrence of infections.[1] About one third of all stroke patients develop infections mostly involving the respiratory and the urinary tract.[2] Post-stroke infections were typically explained as a result of neurological deficits such as impaired protective reflexes,[3] dysphagia,[4] and decreased consciousness, or as treatment associated complications related to mechanical ventilation or indwelling urinary catheters.[4,5] More recent evidence suggests that brain injury itself induces a disturbance of the normally well balanced brainimmune interactions resulting in an immunodepressive status with subsequent infections.[6] The underlying mechanisms of the so called stroke or CNS injury induced immunosuppression (CIDS) are not yet fully understood. It was assumed that the activation of the sympathetic nervous system (SNS) and the hypothalamic-pituitary-adrenal (HPA) axis by the stroke causes a downregulation of immune responses by the end products catecholamines and glucocorticoids.[6] A damage of the SNS and HPA axis structures in the brain by cerebral ischemia could be the trigger of the disinhibition of the autonomic nervous system. Support for this concept came from studies that reported localization-dependent effects of neural-immune interactions after cerebral injury.[7] However, no particular brain region was clearly associated with a higher infection frequency after focal cerebral ischemia. Another concept includes that the activation of the SNS and the HPA axis is initiated by cytokine release of the ischemic tissue and therefore lesion size is more relevant for the development of an immunosuppressive status after stroke than the lesion location.[8,9] Beyond scientific interests a better understanding of the underlying mechanisms of CIDS may also be of clinical relevance, since the modulation of immune responses post-stroke was assumed as a therapeutical target in stroke treatment.[10]

The purpose of our study was: (1) To identify brain regions that might be related to poststroke infection, and (2) to determine whether lesion size is an independent risk factor for infections. 


\section{MATERIALS AND METHODS}

\section{Data Collection}

We enrolled 792 consecutive patients admitted to our certified comprehensive stroke unit [11,12] between December 2004 and December 2006. Only patients with ischemic stroke and intracerebral hemorrhage were included. Patients with transient neurological deficits were included in case brain imaging identified a new ischemic or hemorrhagic lesion consistent with clinical presentation. Patients with sinus thrombosis, secondary intracerebral hemorrhage, and patients with subarachnoid hemorrhage were not included, as well as patients who had an infection at admission or patients who developed an infection within the first 24 hours after admission. Patients were under observation regarding the predefined endpoints until discharge from hospital. All patients or their proxies gave informed consent for data collection. As the study was purely observational, no ethics committee was involved.

\section{Endpoints}

Infections were divided into 3 groups: Pneumonia, urinary tract infection (UTI) and other infections. Infections were diagnosed based on modified Centers for Disease Control and Prevention (CDC) criteria by trained and experienced clinicians.[13]

The diagnosis of pneumonia required 3 or more of the following characteristics: Pulmonary infiltrates in chest radiography, fever $\left(>38.0^{\circ} \mathrm{C}\right)$, dyspnea, abnormal respiratory examination, and white blood cell count $(\mathrm{WBC})>12000 \mathrm{cell} / \mathrm{mL}$.[14]

UTI was defined as fulfilling 3 or more of the following criteria: Lower urinary tract symptoms, positive urine culture for an uropathogen $\left(>10^{5}\right.$ colony-forming units), fever $\left(>38.0^{\circ} \mathrm{C}\right)$, and WBC $>12.000 \mathrm{cell} / \mathrm{mL} .[14]$

Fever $\left(>38.0^{\circ} \mathrm{C}\right)$ in combination with a WBC $>12.000 \mathrm{cell} / \mathrm{mL}$ without fulfilling the criteria of pneumonia or urinary tract infection were defined as other infections.

Neuroimaging studies were performed on admission (computed tomography, CT) and repeated 24-48 hours after admission (magnetic resonance imaging - diffusion weighted imaging $69.1 \%$ or CT $30.9 \%$ ) to confirm lesion size and localization. Lesion size was categorized into 3 groups by the largest diameter: 1 : Lesion $<1.5 \mathrm{~cm}, 2$ : Size between 1.5 
$\mathrm{cm}$ and $1 / 3$ of the middle cerebral artery (MCA) territory or $1.5-5.0 \mathrm{~cm}$, and $3:>1 / 3$ of the MCA territory or $>5.0 \mathrm{~cm}$. Stroke location was categorized by the affected vascular territory. For an additional analysis, we applied a rough classification of stroke location as right and left hemisphere, and excluded patients with bi-hemispheric stroke locations.

\section{Independent variables}

Data on age, sex and other risk factors including arterial hypertension, coronary heart disease, chronic heart failure, atrial fibrillation, diabetes, hyperlipidemia, current smoking status, chronic obstructive pulmonary disease, previous stroke and previous myocardial infarction were obtained from the patient, the relatives, or the patients' general practitioner.

Stroke severity on admission was determined using the National Institutes of Health Stroke Scale (NIHSS). Stroke etiology was classified according to the TOAST criteria. We kept records whether patients received thrombolytic therapy. Orotracheal intubation was recorded if present during the study period.

A fiberoptic endoscopic evaluation of swallowing (FEES) was carried out in a subgroup of patients. Following our in-house guidelines of stroke management, patients had to have either a NIHSS $\geq 3$ points or had to present with a central facial palsy or dysarthria to be eligible for endoscopic evaluation. The examination was carried out as described in detail elsewhere and a 6-point dysphagia score was applied.[15] Patients with a NIHSS $\leq 3$, without central facial palsy, without dysarthria and without clinical evidence of dysphagia as examined in clinical dysphagia screening by the ward physician received a dysaphagia score of 1 .

\section{Statistical analyses}

In single factor analysis possible confounders as age, sex and cardiovascular risk factors were correlated with the predefined endpoints. Categorical variables were tested by Pearson's test or by Fisher's exact test in case of contingency tables containing fewer than five cases. Fulfillment of or deviation from normal distribution of continuous variables was tested by Kolmogorov-Smirnov statistics. Normally distributed continuous variables were compared with Student's $t$ test. The Mann-Whitney $U$ test was used as analogue 
nonparametric test. Chi-square statistics (Fisher's exact test) were also used to determine the level of significance of the Crude odds ratios (cOR) comparing lesion size/location and infection frequency. Logistic regression models were used to adjust for age, sex and all covariates showing significance at $\mathrm{P}<0.05$ in single factor analysis, thus assessing the relative and independent contribution of variables on the infection frequency (adjusted Odds ratio, aOR). For regression analyses we used an interactive backward elimination method based on the likelihood ratio test. The analyses were carried out using the Statistical Package of Social Sciences (version 15.0, SPSS Inc., Chicago, IL).

\section{RESULTS}

\section{Baseline data}

Five hundred ninety-one of the 792 patients admitted to our stroke during the study period had an ischemic stroke or a primary intracerebral hemorrhage and were included in the study. One hundred sixty-six patients had a non-ischemic cause of stroke, subarachnoid hemorrhage, sinus thrombosis, or secondary intracerebral hemorrhage and were therefore excluded. Due to an incomplete NIHSS 24 patients were excluded. Eleven patients had an infection on admission and were therefore excluded. A dysphagia score was determined in 370 patients. Patients were under observation for a mean duration of 14.2 days (SD 11). Demographics and baseline characteristics are given in table 1.

Pneumonia occurred in 72 (12.2\%) patients, 65 patients (11.0\%) had an UTI and $100(16.9)$ patients had other infections.

The following factors were associated with the detected infections in single factor analysis (Table 1): Pneumonia was associated with higher age, COPD and thrombolysis. Male gender was less common in patients with UTI and other infections. NIHSS was higher in patients with any infection. Dysphagia and mechanical ventilation were more common in patients with pneumonia and other infections. The level of consciousness was associated with pneumonia and UTI. 
Stroke etiology was also significantly associated with the frequency of infections: Intracerebral hemorrhage was more frequent in patients with pneumonia and other infections. Lacunar stroke, stroke of other etiology and stroke of undeclared etiology were less frequent in patients with pneumonia. Stroke of undeclared etiology was also less common in patients with other infections.

\section{Impact of lesion location on infection frequency}

Results of the univariate analysis are detailed in table 2. Regarding different stroke locations only strokes in the left anterior cerebral artery territory were associated with pneumonia (cOR 3.8, $\mathrm{P}<0.05)$. No other lesion location showed a statistically significant association with any infection. After adjustment for possible confounders in logistic regression analyses, the significant association between left anterior cerebral artery infarction and pneumonia disappeared (aOR 2.2, $\mathrm{P}=0.196)$. In addition, we analyzed whether patients with a left or patients with a right sided stroke had more infections. We found no statistical significant association of stroke lateralization with the frequency of pneumonia, UTI or other infections. Impact of lesion size on infection frequency

Small lesion size $(<1.5 \mathrm{~cm})$ was significantly associated with a lower frequency of pneumonia (cOR 0.2, $\mathrm{P}<0.001)$ and UTI (cOR 0.4, $\mathrm{P}<0.01)$. The largest stroke size $(>5.0 \mathrm{~cm}$ or $>1 / 3$ of the MCA territory) was positively associated with pneumonia (cOR 7.4, $\mathrm{P}<0.001$ ), UTI (cOR 1.9, $\mathrm{P}<0.05)$ and other infections (cOR 2.3, $\mathrm{P}<0.01)$ (table 3).

After adjustment for covariates the smallest stroke size was negatively correlated with the occurrence of UTI (aOR 0.4, $\mathrm{P}<0.01)$ and pneumonia (aOR $0.5, \mathrm{P}<0.05)$. The largest lesions were associated with pneumonia (aOR 3.5, $\mathrm{P}<0.001)$ (figure 1).

Since dysphagia was associated with pneumonia (Table 1) we performed an analysis including only patients in which a dysphagia score was determined $(n=370)$. The effect of the largest stroke size regarding the frequency of pneumonia remained significant (aOR 3.9, $\mathrm{P}<0.01$ ). The association between small lesions and the occurrence of pneumonia was no longer significant (aOR 0.69, $\mathrm{P}=0.49)$. 


\section{DISCUSSION}

\section{Stroke size and stroke location as predictors of infection}

Stroke severity was shown to be crucial for the development of complications in post-stroke patients.[16,17] In our study lesion size had a significant impact on post-stroke infection frequency beyond stroke severity. Patients with larger strokes were at higher risk for pneumonia (aOR 3.5, $\mathrm{P}<0.001$ ) compared to those with a smaller stroke. Patients with a small lesion had a lower risk for UTI (aOR 0.4, P<0.01) compared to those with a larger lesion. So far, only limited data are available on the impact of lesion size on the occurrence of post-stroke infections.[2,4,18] Previous studies analyzed smaller samples of patients and associations between stroke size and the risk for infection were not adjusted for relevant covariates.[2,4,18] In our study, we were able to show that the effect of lesion size on the frequency of any infection is independent of strong predictors such as age, gender, NIHSS, level of consciousness, mechanical ventilation or dysphagia.

We only found the left anterior cerebral artery (ACA) territory to be associated with the occurrence of pneumonia (cOR 3.8, $\mathrm{P}<0.05$ ). However, after adjustment for confounders this association was not statistically significant anymore (aOR 2.2, $\mathrm{P}=0.196)$. Other studies found total anterior circulation infarcts (TACl) as defined by the Oxfordshire Community Stroke Project (OCSP) and non-lacunar basal-ganglia infarctions to be significantly associated with post-stroke infections.[18-21] The term TACI describes no distinct infarct location.[20] It defines a clinical syndrome consisting of the combination of higher cerebral dysfunction (eg, aphasia, visuospatial disorder); homonymous visual field defect; and ipsilateral motor and/or sensory deficit of at least two areas of the face, arm, and leg.[20] The significant association between non-lacunar basal-ganglia infarctions and pneumonia in the study of Walter et al. has to be critically evaluated due to a very small number of patients $(n=8)$ showing this infarct location and a rather small study population of 236 patients.[18]

Besides the impact of particular infarct locations on post-stroke infections we investigated the effect of stroke lateralization on infection frequency. We found no association between lesion lateralization and the occurrence of any of the detected infections. In contrast to our 
finding, left sided stroke was considered as a risk factor for infectious disease by Koch et al.[22] However, their results are limited because no infections as defined by predetermined criteria were detected but only WBC and C-reactive protein (CRP) were measured within the first 24 hours after symptom onset, which is a very short observation interval. In addition, this study included a small number of patients and the result of higher WBC and elevated CRP was not adjusted for relevant confounders such as NIHSS or age.

\section{Scientific and clinical relevance of associations between lesion size and location with post-stroke infection}

The finding that stroke size is an independent risk factor for the occurrence of post-stroke infections, whereas there is no association with the affected vascular territory might give some insights into the underlying mechanisms of post-stroke infections. As reviewed elsewhere the activation of the SNS and the HPA axis seems to be of particular importance for the development of CIDS.[23] The primary trigger of this activation is, however, unknown so far. It was postulated, that cytokine production in the brain after ischemia stimulates the HPA axis and the SNS.[8,9] Our finding that lesion size is an independent risk factor for post-stroke infections offers some support to this hypothesis since a larger lesion might release a higher amount of cytokines and thereby aggravates immunodepression. However, this conclusion is limited, because our study is restricted to the detection of the endpoint infection and not the intermediate steps such as cerebral cytokine production and plasma cortisol or catecholamine levels.

A better understanding of the pathophysiology of CIDS is certainly of clinical relevance. Prophylactic antibiotic therapy after stroke was assumed to prevent post-stroke infections and thereby reduce mortality. Exclusion of patients with small infarcts or lacunar strokes as done in a recent clinical trial might enhance the benefit of a preventive antibacterial therapy.[24] In addition, interventions with drugs that target inflammatory responses poststroke need a wide knowledge of brain immune interactions. Currently, trials of candidate stroke drugs that interfere with the immune system, such as the granulocyte-colony stimulating factor (G-CSF) or albumin are underway.[10,25] 


\section{Methodologic considerations}

Our study has several strengths. By using numerous brain areas we were able to separate the impact of lesion size from location effects. The diagnosis of infections was based on clinically accepted predetermined criteria. In addition, all known relevant factors for the development of post-stroke infections were included in the logistic regression analysis. Moreover, an instrumental investigation of swallowing impairment was performed in a reasonable number of patients. Furthermore, the observational time was fairly long (mean 14.2 days). A potential weakness of our study might be the fact, that brain regions were defined by vascular territory instead of anatomically defined brain regions, which are of particular importance for autonomic nervous system regulation. However, our study was designed to be as clinically relevant as possible. Stroke locations were therefore described by the affected vascular territory which is a common mode in stroke medicine. Despite the fairly large number of patients included in our study we can not rule out that the lack of a statistical significant association between stroke location and infection might be caused by the relatively small number of strokes in several regions such as the anterior cerebral artery territory or the brain stem.

\section{Conclusion}

We found that infections after stroke are independently associated with stroke size. A particular brain region predicting post-stroke infections could not be identified. These findings contribute to a better knowledge of the development of post-stroke infections. 
Acknowledgments

None

Competing interests

None

Funding

None 


\section{Reference List}

(1) Ovbiagele B, Hills NK, Saver JL, et al. Frequency and determinants of pneumonia and urinary tract infection during stroke hospitalization. J Stroke Cerebrovasc Dis 2006;15(5):209-213.

(2) Vargas M, Horcajada JP, Obach V, et al. Clinical consequences of infection in patients with acute stroke: is it prime time for further antibiotic trials? Stroke 2006;37(2):461-465 .

(3) Nakajoh K, Nakagawa T, Sekizawa K, et al. Relation between incidence of pneumonia and protective reflexes in post-stroke patients with oral or tube feeding. J Intern Med 2000;247(1):39-42.

(4) Hilker R, Poetter C, Findeisen N, et al. Nosocomial pneumonia after acute stroke: implications for neurological intensive care medicine. Stroke 2003;34(4):975-981.

(5) Ersoz M, Ulusoy H, Oktar MA, et al. Urinary tract infection and bacteriuria in stroke patients: frequencies, pathogen microorganisms, and risk factors. Am J Phys Med Rehabil 2007;86(9):734-741.

(6) Dirnagl U, Klehmet J, Braun JS, et al. Stroke-induced immunodepression: experimental evidence and clinical relevance. Stroke 2007;38(2 Suppl):770-773.

(7) Meador KJ, Loring DW, Ray PG, et al. Role of cerebral lateralization in control of immune processes in humans. Ann Neurol 2004;55(6):840-844.

(8) Woiciechowsky C, Schoning B, Lanksch WR, et al. Mechanisms of brain-mediated systemic anti-inflammatory syndrome causing immunodepression. $J$ Mol Med 1999;77(11):769-780.

(9) Fassbender K, Schmidt R, Mossner R, et al. Pattern of activation of the 
hypothalamic-pituitary-adrenal axis in acute stroke. Relation to acute confusional state, extent of brain damage, and clinical outcome. Stroke 1994;25(6):1105-1108.

(10) Minnerup J, Schabitz WR. Multifunctional actions of approved and candidate stroke drugs. Neurotherapeutics 2009;6(1):43-52.

(11) Alberts MJ, Latchaw RE, Selman WR, et al. Recommendations for comprehensive stroke centers: a consensus statement from the Brain Attack Coalition. Stroke 2005;36(7):1597-1616.

(12) Leys D, Ringelstein EB, Kaste M, et al. The main components of stroke unit care: results of a European expert survey. Cerebrovasc Dis 2007;23(5-6):344-352.

(13) Garner JS, Jarvis WR, Emori TG, et al. CDC definitions for nosocomial infections, 1988. Am J Infect Control 1988;16(3):128-140.

(14) Definition nosokomialer Infektionen (CDC-Definitionen). Robert Koch-Institut Berlin 2005.

(15) Dziewas R, Warnecke T, Olenberg S, et al. Towards a basic endoscopic assessment of swallowing in acute stroke - development and evaluation of a simple dysphagia score. Cerebrovasc Dis 2008;26(1):41-47.

(16) Langhorne P, Stott DJ, Robertson L, et al. Medical complications after stroke: a multicenter study. Stroke 2000;31(6):1223-1229.

(17) Spratt N, Wang Y, Levi C, et al. A prospective study of predictors of prolonged hospital stay and disability after stroke. J Clin Neurosci 2003;10(6):665-669.

(18) Walter U, Knoblich R, Steinhagen V, et al. Predictors of pneumonia in acute stroke patients admitted to a neurological intensive care unit. J Neurol 2007;254(10):1323- 
1329.

(19) Aslanyan S, Weir CJ, Diener HC, et al. Pneumonia and urinary tract infection after acute ischaemic stroke: a tertiary analysis of the GAIN International trial. Eur J Neurol 2004;11(1):49-53.

(20) Bamford J, Sandercock P, Dennis M, et al. Classification and natural history of clinically identifiable subtypes of cerebral infarction. Lancet 1991;337(8756):15211526.

(21) Sellars C, Bowie L, Bagg J, et al. Risk factors for chest infection in acute stroke: a prospective cohort study. Stroke 2007;38(8):2284-2291 .

(22) Koch HJ, Uyanik G, Bogdahn U, et al. Relation between laterality and immune response after acute cerebral ischemia. Neuroimmunomodulation 2006;13(1):8-12.

(23) Meisel C, Schwab JM, Prass K, et al. Central nervous system injury-induced immune deficiency syndrome. Nat Rev Neurosci 2005;6(10):775-786.

(24) Harms H, Prass K, Meisel C, et al. Preventive antibacterial therapy in acute ischemic stroke: a randomized controlled trial. PLOS ONE 2008;3(5):e2158.

(25) Bath PM, Sprigg N. Colony stimulating factors (including erythropoietin, granulocyte colony stimulating factor and analogues) for stroke. Cochrane Database Syst Rev 2007;(2):CD005207. 
TABLE 1. Main characteristics of study population

\begin{tabular}{|c|c|c|c|c|}
\hline Patient characteristics & Total & Pneumonia & $\begin{array}{l}\text { Urinary tract } \\
\text { infection }\end{array}$ & $\begin{array}{c}\text { Other } \\
\text { infections }\end{array}$ \\
\hline All, n (\%) & 591 & $72(12)$ & $65(11)$ & $100(17)$ \\
\hline Age, mean (SD) & $67.7(13.8)$ & $71.2(12.3)^{*}$ & $69.8(14.9)$ & $66.3(15.0)$ \\
\hline Male, n (\%) & 325 & $43(60)$ & $24(37) \dagger$ & $44(44)^{\star}$ \\
\hline \multirow{2}{*}{ NIHSS $\begin{array}{l}\text { mean (SD) } \\
\text { median (IQR) }\end{array}$} & $6.9(6.0)$ & $12.4(6.7) \ddagger$ & $8.9(5.4) \dagger$ & $8.7(6.7) \dagger$ \\
\hline & $5(2-11)$ & $13(8-16)$ & $8(4-14)$ & $7(3-13)$ \\
\hline Level of consciousness & & $\ddagger$ & $\dagger$ & \\
\hline Alert & $486(82)$ & $41(57)$ & $44(68)$ & $74(74)$ \\
\hline Drowsy/Stupor & $90(15)$ & $24(33)$ & $20(31)$ & $22(22)$ \\
\hline Coma & $15(3)$ & $7(10)$ & $1(2)$ & $4(4)$ \\
\hline Hypertension, n (\%) & $423(72)$ & $52(72)$ & $45(69)$ & $68(68)$ \\
\hline Hyperlipidemia, n (\%) & $136(23)$ & $11(15)$ & $9(13)$ & $19(19)$ \\
\hline Diabetes mellitus, $\mathrm{n}(\%)$ & $114(19)$ & $17(24)$ & $13(20)$ & $14(14)$ \\
\hline Chronic heart failure, $\mathrm{n}(\%)$ & $36(6)$ & $5(7)$ & $4(6)$ & $5(5)$ \\
\hline Atrial fibrillation, $\mathrm{n}(\%)$ & $121(20)$ & $21(29)$ & $15(23)$ & $22(22)$ \\
\hline Smoking, n (\%) & $119(20)$ & $10(14)$ & $11(17)$ & $21(21)$ \\
\hline COPD, n (\%) & $35(6)$ & $9(13)^{*}$ & $4(6)$ & $6(6)$ \\
\hline Previous myocardial infarction, $\mathrm{n}(\%)$ & $39(7)$ & $5(7)$ & $3(5)$ & $6(6)$ \\
\hline Previous stroke, n (\%) & $117(20)$ & $11(15)$ & $14(22)$ & $16(16)$ \\
\hline Thrombolysis, n (\%) & $110(19)$ & $25(35) \dagger$ & $10(14)$ & $24(24)$ \\
\hline Mechanical ventilation, n (\%) & $70(12)$ & $34(47) \ddagger$ & $8(11)$ & $19(19)^{\star}$ \\
\hline Dysphagia Score, n (\%) & $370(63)$ & $39(54)$ & $34(52)$ & $62(62)$ \\
\hline Dysphagia Score, mean (SD) & $1.9(1.6)$ & $4.2(1.8) \ddagger$ & $2.3(1.6)$ & $2.9(1.9) \ddagger$ \\
\hline \multicolumn{5}{|l|}{ Etiology } \\
\hline Atherothrombotic, n (\%) & $123(21)$ & $18(25)$ & $11(17)$ & $20(20)$ \\
\hline Cardioembolic, n (\%) & $198(34)$ & $31(43)$ & $25(38)$ & $36(36)$ \\
\hline Lacunar stroke, n (\%) & $55(9)$ & $1(1) \dagger$ & $2(3)$ & $4(4)$ \\
\hline Undetermined etiology, $\mathrm{n}(\%)$ & $102(17)$ & $6(8) \dagger$ & $13(20)$ & $10(10)^{*}$ \\
\hline Other etiology, $n(\%)$ & $58(10)$ & $2(3) \dagger$ & $4(6)$ & $15(15)$ \\
\hline Intracerebral hemorrhage, $\mathrm{n}(\%)$ & $55(9)$ & $14(19) \dagger$ & $10(14)$ & $15(15)^{\star}$ \\
\hline
\end{tabular}

${ }^{*} p<0.05, \dagger p<0.01, \neq p<0.001$

$P$-values indicate the level of significance of the association of infection frequency with the listed variables. Categorical variables were tested by Pearson's test or Fisher's exact test. Continuous variables were compared with Student's t test and Mann-Whitney U test respectively. IQR, Interquartile Range. 
TABLE 2. Stroke Location and infection frequency

\begin{tabular}{|c|c|c|c|c|c|c|c|}
\hline \multirow[t]{2}{*}{ Location } & \multirow{2}{*}{$\begin{array}{c}\text { Total } \\
\mathrm{n}\end{array}$} & \multicolumn{2}{|c|}{$\begin{array}{c}\text { Pneumonia } \\
\mathrm{n}=72\end{array}$} & \multicolumn{2}{|c|}{$\begin{array}{l}\text { Urinary tract } \\
\text { infection } \\
n=65\end{array}$} & \multicolumn{2}{|c|}{$\begin{array}{c}\text { Other } \\
\text { infections } \\
n=100\end{array}$} \\
\hline & & $\mathrm{n}$ & $\mathrm{cOR}$ & $\mathrm{n}$ & $\mathrm{cOR}$ & $\mathrm{n}$ & $\mathrm{cOR}$ \\
\hline ACA, r. (\%) & 20 & $5(25)$ & 2.5 & 0 & & $5(25)$ & 1.7 \\
\hline ACA, I. (\%) & 18 & $6(33)$ & $3.8^{*}$ & 0 & & $2(11)$ & 0.6 \\
\hline ACA, bilat. (\%) & 3 & $1(33)$ & 3.6 & 0 & & $3(100)$ & \\
\hline Basal ganglia, r. (\%) & 110 & $17(16)$ & 1.4 & $13(12)$ & 1.1 & $20(18)$ & 1.1 \\
\hline Basal ganglia, I. (\%) & 135 & $18(13)$ & 1.1 & $18(13)$ & 1.3 & $30(22)$ & 1.6 \\
\hline Basal ganglia, bilat. (\%) & 1 & 0 & & 0 & & 0 & \\
\hline MCA cortical, r. (\%) & 137 & $23(17)$ & 1.7 & $18(13)$ & 1.3 & $23(17)$ & 1.0 \\
\hline MCA cortical, I. (\%) & 161 & $19(12)$ & 1.0 & $22(14)$ & 1.4 & $30(19)$ & 1.2 \\
\hline MCA corical, bilat. (\%) & 6 & $1(17)$ & 1.4 & 0 & & $3(50)$ & 5.0 \\
\hline PCA cortical, r. (\%) & 27 & $1(4)$ & 0.3 & 0 & & $7(26)$ & 1.8 \\
\hline PCA cortical, I. (\%) & 30 & $3(10)$ & 0.8 & $6(20)$ & 2.1 & $5(17)$ & 1.0 \\
\hline PCA cortical, bilat. (\%) & 8 & $1(13)$ & 1.0 & 0 & & $1(13)$ & 0.7 \\
\hline Thalamus, r. (\%) & 27 & $5(19)$ & 1.7 & $2(7)$ & 0.6 & $5(19)$ & 1.1 \\
\hline Thalamus, I. (\%) & 31 & $3(10)$ & 0.8 & $4(13)$ & 1.2 & $4(13)$ & 0.7 \\
\hline Thalamus, bilat. (\%) & 8 & $1(13)$ & 1.0 & 0 & & $2(25)$ & 1.7 \\
\hline Brainstem, r. (\%) & 21 & $2(10)$ & 0.8 & $3(14)$ & 1.4 & $3(14)$ & 0.8 \\
\hline Brainstem, I. (\%) & 25 & $3(12)$ & 1.0 & $2(8)$ & 0.7 & $1(4)$ & 0.2 \\
\hline Brainstem, bilat. (\%) & 6 & $2(33)$ & 3.7 & 0 & & $1(17)$ & 1.0 \\
\hline Cerebellum, r. (\%) & 30 & $4(13)$ & 1.1 & $4(13)$ & 1.3 & $3(10)$ & 0.5 \\
\hline Cerebellum, I. (\%) & 19 & $3(16)$ & 1.4 & $1(5)$ & 0.4 & $6(32)$ & 2.4 \\
\hline Cerebellum, bilat. (\%) & 7 & $2(29)$ & 2.9 & 0 & & $1(14)$ & 0.8 \\
\hline
\end{tabular}

I., left; r., right.; bilat., bilateral; $A C A$, anterior cerebral artery territory;

MCA, middle cerebral artery territory; PCA, posterior cerebral artery territory;

cOR, crude Odds ratio 
TABLE 3. Lesion size and infection, logistic regression models

\begin{tabular}{|c|c|c|c|c|c|c|c|c|c|c|}
\hline \multirow[t]{2}{*}{ Lesion size } & \multirow{2}{*}{$\begin{array}{c}\begin{array}{c}\text { Total } \\
\mathrm{n}=594 \\
\mathrm{n}\end{array} \\
\end{array}$} & \multicolumn{3}{|c|}{$\begin{array}{l}\text { Pneumonia } \\
n=72\end{array}$} & \multicolumn{3}{|c|}{$\begin{array}{l}\text { Urinary tract infection } \\
\qquad \mathrm{n}=65\end{array}$} & \multicolumn{3}{|c|}{$\begin{array}{l}\text { Other infections } \\
\quad \mathrm{n}=100\end{array}$} \\
\hline & & $\mathrm{n}$ & $\mathrm{cOR}$ & $\mathrm{aOR}$ & $n$ & $\mathrm{cOR}$ & $\mathrm{aOR}$ & $\mathrm{n}$ & $\mathrm{cOR}$ & $\mathrm{aOR}$ \\
\hline$<1.5 \mathrm{~cm} \mathrm{( \% )}$ & 281 & $14(5)$ & $0.2 \ddagger$ & $0.5^{*}$ & $19(7)$ & $0.4 \dagger$ & $0.4 \dagger$ & $39(14)$ & 0.7 & 0.9 \\
\hline $\begin{array}{l}1.5 \text { to } 5.0 \mathrm{~cm} \text { or } 1.5 \\
\mathrm{~cm} \text { to } 1 / 3 \mathrm{MCA}(\%)\end{array}$ & 185 & $16(9)$ & 0.6 & 0.6 & $25(14)$ & 1.4 & 1.4 & $27(15)$ & 0.8 & 0.8 \\
\hline $\begin{array}{l}>5.0 \mathrm{~cm} \text { or } \\
>1 / 3 \mathrm{MCA}(\%)\end{array}$ & 125 & $\begin{array}{c}42 \\
(34)\end{array}$ & $7.4 \ddagger$ & $3.5 \ddagger$ & $21(17)$ & $1.9^{*}$ & 1.4 & $34(27)$ & $2.3 \dagger$ & 1.7 \\
\hline
\end{tabular}

${ }^{*} \mathrm{p}<0.05, \dagger \mathrm{p}<0.01, \neq \mathrm{p}<0.001$

MCA, middle cerebral artery territory; cOR, crude Odds ratio; aOR, adjusted Odds ratio 


\section{Figure 1}

Infarct size and infection frequency: When adjusted for possible confounders in logistic regression analysis, the smallest stroke size was negatively correlated with UTI (aOR 0.4, $\mathrm{P}<0.01)$ and pneumonia (aOR $0.5, \mathrm{P}<0.05)$ and the largest lesions were associated with the occurrence of pneumonia (aOR 3.5, $\mathrm{P}<0.001$ ). Small: Lesions $<1.5 \mathrm{~cm}$, Middle: Lesion size between $1.5 \mathrm{~cm}$ and $1 / 3$ of the MCA territory or $1.5-5.0 \mathrm{~cm}$, Large: Lesions $>1 / 3$ of the MCA territory or $>5.0 \mathrm{~cm} .{ }^{*} p<0.05,{ }^{* *} p<0.01,{ }^{* *} p<0.001$. 
Adjusted

Odds

Ratios

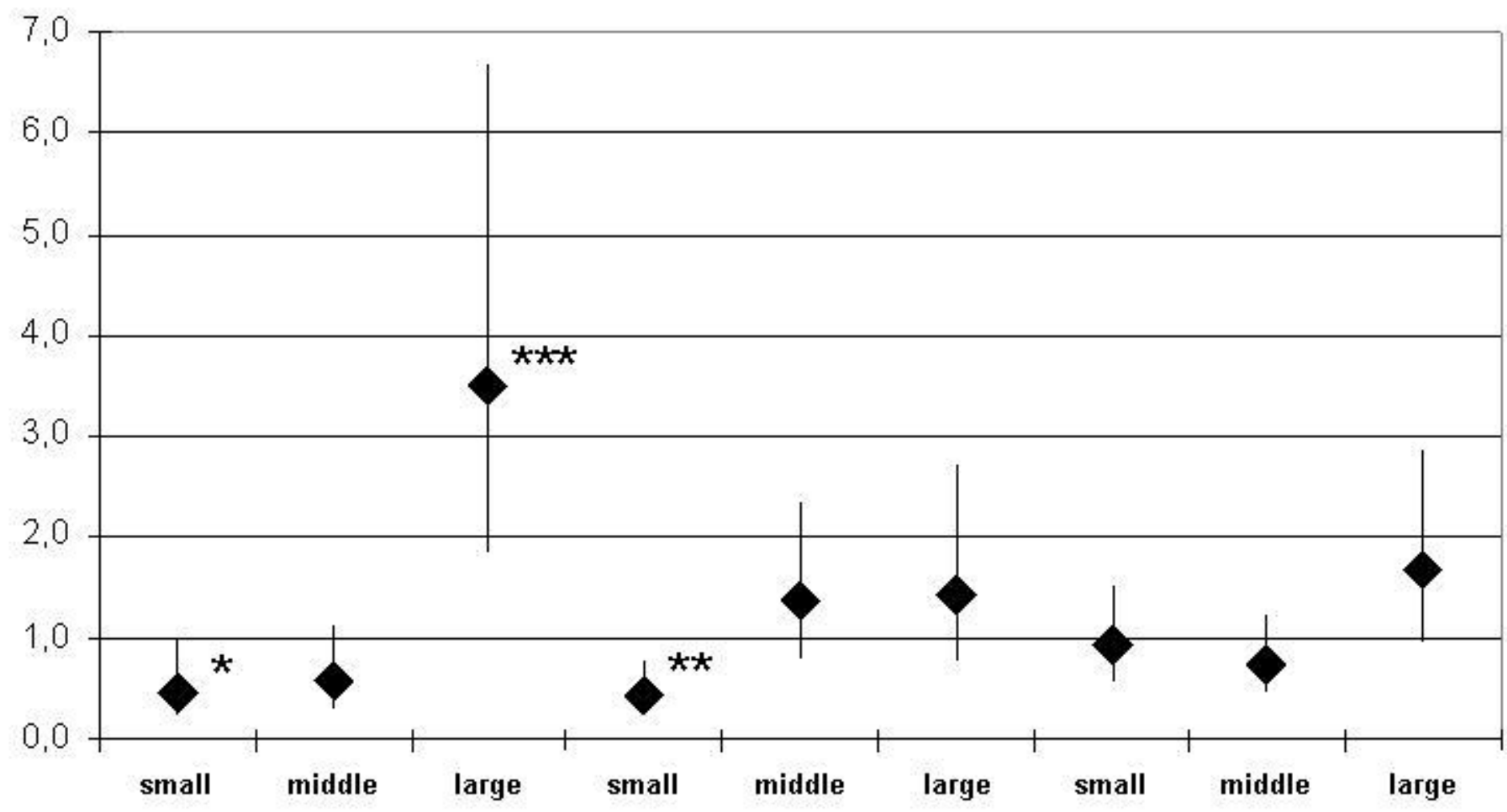

Infarct

Pneumonia

Urinary Tract

Other Infections

size Infections 